\title{
ON HERMITE-HADAMARD TYPE INEQUALITIES FOR INTERVAL-VALUED MULTIPLICATIVE INTEGRALS
}

\author{
Muhammad Aamir ALI ${ }^{1}$, Zhiyue ZHANG ${ }^{1}$, Hüseyin BUDAK ${ }^{2}$, and Mehmet Zeki SARIKAYA 2 \\ ${ }^{1}$ Jiangsu Key Laboratory of NSLSCS, School of Mathematical Sciences, Nanjing Normal \\ University, 210023 CHINA \\ ${ }^{2}$ Department of Mathematics, Faculty of Science and Arts, Düzce University, Düzce TURKEY
}

\begin{abstract}
In this work, we define multiplicative integrals for interval-valued functions. We establish some new Hermite-Hadamard type inequalities in the setting of interval-valued multiplicative calculus and give some examples to illustrate our main results. We also discuss special cases of our main results which are the extension of already established results.
\end{abstract}

\section{INTRODUCTION}

The Hermite-Hadamard inequality discovered by C. Hermite and J. Hadamard, (see [14, 32, pp. 137]) is one of the most well established inequalities in the theory of convex functions with a geometrical interpretation and many applications. These inequalities state that, if $F: I \rightarrow \mathbb{R}$ is a convex function on the interval $I$ of real numbers and $a, b \in I$ with $a<b$, then

$$
F\left(\frac{a+b}{2}\right) \leq \frac{1}{b-a} \int_{a}^{b} F(x) d x \leq \frac{F(a)+F(b)}{2} .
$$

Both inequalities in (1.1) hold in the reversed direction if $F$ is concave. We note that Hermite-Hadamard inequality may be regarded as a refinement of the concept of convexity and it follows easily from Jensen's inequality. Hermite-Hadamard inequality for convex functions has received renewed attention in recent years and a remarkable variety of refinements and generalizations have been studied, see 1 , 2, $7,8,11,15,18,26,31,36,40]$ and reference therein.

2020 Mathematics Subject Classification. 26A09, 26D10, 26D15, 33E20.

Keywords and phrases. Convex functions, logarithmically convex functions, interval-valued functions, multiplicative integral operator and Hermite-Hadamard inequalities.

凶mahr.muhammad.aamir@gmail.com; 05298@njnu.edu.cn-Corresponding author; hsyn.budak@gmail.com; sarikayamz@gmail.com

(D) 0000-0001-5341-4926; 0000-0001-7070-2532; 0000-0001-8843-955X; 0000-0003-3856-6360.

Communications Faculty of Sciences University of Ankara-Series A1 M2020 Ankara University 
On the other hand, interval analysis is a particular case of set-valued analysis which is the study of sets in the spirit of mathematical analysis and general topology. It was introduced as an attempt to handle interval uncertainty that appears in many mathematical or computer models of some deterministic real-world phenomena. An old example of interval enclosure is Archimede's method which is related to compute of the circumference of a circle. In 1966, the first book related to interval analysis was given by Moore who is known as the first user of intervals in computational mathematics, see 27]. After his book, several scientists started to investigate theory and application of interval arithmetic. Nowadays, because of its applications, interval analysis is a useful tool in various area which are interested intensely in uncertain data. You can see applications in computer graphics, experimental and computational physics, error analysis, robotics and many others. What's more, several important inequalities (Hermite-Hadamard, Ostrowski, etc.) have been studied for the interval-valued functions in recent years. In 9, 10, Chalco-Cano et al. obtained Ostrowski type inequalities for interval-valued functions by using Hukuhara derivative for interval-valued functions. In 33], RománFlores et al. established Minkowski and Beckenbach's inequalities for intervalvalued functions. For the others, please see $12,13,19,33,34$. However, inequalities were studied for more general set-valued maps. For example, in 35], Sadowska gave the Hermite-Hadamard inequality. In 21, 41], authors established HermiteHadamard type inequalities for co-ordinated convex interval-valued functions. For the other studies, you can see $3,25,29$.

The main purpose of this paper is to define *integral/multiplicative integral for interval-valued functions and to obtain Hermite-Hadamard inequality via these integrals.

The overall structure of the study takes the form of six sections including introduction. The remainder of this work is organized as follows: we first recall the interval calculus by giving the several definitions and properties in Section 2. In section 3, we define multiplicative integral for interval-valued functions and give some basic properties of this newly define integral. In Section 4, we define logarithmically interval-valued $h$-convex functions and discuss special cases and properties of this class of functions. In section 5, we obtain Hermite-Hadamard inequalities and related inequalities for our new class of convex functions by utilizing our newly define integral. At the end, in section 6, we give concluding remarks about our work.

\section{Interval Calculus}

A real valued interval $X$ is bounded, closed subset of $\mathbb{R}$ defined by

$$
X=[\underline{X}, \bar{X}]=\{t \in \mathbb{R}: \underline{X} \leq t \leq \bar{X}\}
$$

where $\underline{X}, \bar{X} \in \mathbb{R}$ and $\underline{X} \leq \bar{X}$. The numbers $\underline{X}$ and $\bar{X}$ are called the left and the right endpoints of interval $X$, respectively. When $\bar{X}=\underline{X}=a$, the interval $X$ is 
said to be degenerate and we use the form $X=a=[a, a]$. Also, we call $X$ positive if $\underline{X}>0$ or negative if $\bar{X}<0$. The set of all closed intervals of $\mathbb{R}$, the sets of all closed positive intervals of $\mathbb{R}$ and closed negative intervals of $\mathbb{R}$ is denoted by $\mathbb{R}_{\mathcal{I}}$, $\mathbb{R}_{\mathcal{I}}^{+}$and $\mathbb{R}_{\mathcal{I}}^{-}$respectively. The Hausdorff-Pompeiu distance between the intervals $X$ and $Y$ is defined by

$$
d(X, Y)=d([\underline{X}, \bar{X}],[\underline{Y}, \bar{Y}])=\max \{|\underline{X}-\underline{Y}|,|\bar{X}-\bar{Y}|\} .
$$

It is known that $\left(\mathbb{R}_{\mathcal{I}}, d\right)$ is a complete metric space [4].

Now, we give the definitions of basic interval arithmetic operations for the intervals $X$ and $Y$ as follows:

$$
\begin{aligned}
X+Y & =[\underline{X}+\underline{Y}, \bar{X}+\bar{Y}], \\
X-Y & =[\underline{X}-\bar{Y}, \bar{X}-\underline{Y}], \\
X . Y & =[\min S, \max S] \text { where } S=\{\underline{X} \underline{Y}, \underline{X} \bar{Y}, \bar{X} \underline{Y}, \bar{X} \bar{Y}\}, \\
X / Y & =[\min T, \max T] \text { where } T=\{\underline{X} / \underline{Y}, \underline{X} / \bar{Y}, \bar{X} / \underline{Y}, \bar{X} / \bar{Y}\} \text { and } 0 \notin Y .
\end{aligned}
$$

Scalar multiplication of the interval $X$ is defined by

$$
\lambda X=\lambda[\underline{X}, \bar{X}]= \begin{cases}{[\lambda \underline{X}, \lambda \bar{X}],} & \lambda>0 \\ \{0\}, & \lambda=0 \\ {[\lambda \bar{X}, \lambda \underline{X}],} & \lambda<0 .\end{cases}
$$

where $\lambda \in \mathbb{R}$.

The opposite of the interval $X$ is

$$
-X:=(-1) X=[-\bar{X},-\underline{X}]
$$

for $\lambda=-1$.

The subtraction is given by

$$
X-Y=X+(-Y)=[\underline{X}-\bar{Y}, \bar{X}-\underline{Y}] .
$$

Use of monotonic functions

$$
F(X)=[F(\underline{X}), F(\bar{X})] .
$$

For example, $F(x)=e^{x}, x \in \mathbb{R}$ and $F(x)=\ln x, x>0$ then we have

$$
\begin{aligned}
\exp (X) & =[\exp (\underline{X}), \exp (\bar{X})] \\
\ln (X) & =[\ln (\underline{X}), \ln (\bar{X})] .
\end{aligned}
$$

In general, $-X$ is not additive inverse for $X$ i.e $X-X \neq 0$. 
The definitions of operations lead to a number of algebraic properties which allows $\mathbb{R}_{\mathcal{I}}$ to be quasilinear space (see, 24]). They can be listed as follows (see, [4, 22, 24 , 27 ):

(1) (Associativity of addition) $(X+Y)+Z=X+(Y+Z)$ for all $X, Y, Z \in \mathbb{R}_{\mathcal{I}}$,

(2) (Additive element) $X+0=0+X=X$ for all $X \in \mathbb{R}_{\mathcal{I}}$,

(3) (Commutativity of addition) $X+Y=Y+X$ for all $X, Y \in \mathbb{R}_{\mathcal{I}}$,

(4) (Cancelation law) $X+Z=Y+Z \Longrightarrow X=Y$ for all $X, Y, Z \in \mathbb{R}_{\mathcal{I}}$,

(5) (Associativity of multiplication) $(X . Y) \cdot Z=X .(Y . Z)$ for all $X, Y, Z \in \mathbb{R}_{\mathcal{I}}$,

(6) (Commutativity of multiplication) $X . Y=Y . X$ for all $X, Y \in \mathbb{R}_{\mathcal{I}}$,

(7) (Unit element) $X .1=1 . X$ for all $X \in \mathbb{R}_{\mathcal{I}}$,

(8) (Associate law) $\lambda(\mu X)=(\lambda \mu) X$ for all $X \in \mathbb{R}_{\mathcal{I}}$ and all $\lambda, \mu \in \mathbb{R}$,

(9) (First distributive law) $\lambda(X+Y)=\lambda X+\lambda Y$ for all $X, Y \in \mathbb{R}_{\mathcal{I}}$ and all $\lambda \in \mathbb{R}$,

(10) (Second distributive law) $(\lambda+\mu) X=\lambda X+\mu X$ for all $X \in \mathbb{R}_{\mathcal{I}}$ and all $\lambda, \mu \in \mathbb{R}$. Besides these properties, the distributive law is not always valid for intervals. For example, $X=[1,2], Y=[2,3], Z=[-2,-1]$

$$
X .(Y+Z)=[0,4]
$$

whereas

$$
X . Y+X . Z=[-2,5] .
$$

But, this law hold in certain cases. If $Y Z>0$, then

$$
X .(Y+Z)=X . Y+X . Z \text {. }
$$

What's more, one of the set property is the inclusion " $\subseteq$ " that is given by

$$
X \subseteq Y \Longleftrightarrow \underline{Y} \leq \underline{X} \text { and } \bar{X} \leq \bar{Y} \text {. }
$$

Considering together with arithmetic operations and inclusion, one has the following property which is called inclusion isotony of interval operations:

Let $\odot$ be the addition, multiplication, subtraction or division. If $X, Y, Z$ and $T$ are intervals such that

$$
X \subseteq Y \text { and } Z \subseteq T
$$

then the following relation is valid

$$
X \odot Z \subseteq Y \odot T .
$$

\section{3. *Integral of Interval-Valued Functions}

In this section, we define *integral or multiplicative integral for the interval-valued functions and give properties of this new integral. Throughout in this section, we shall use $F(t)=[\underline{F}(t), \bar{F}(t)]$ is positive interval-valued function, $I R$ is the notation for the interval-valued integrals and $I^{*}$ means the multiplicative integral.

First, we recall that the concept of *integral is denoted by $\int_{a}^{b}(F(x))^{d x}$ which introduced by Bashirov et al. in [5. In multiplicative integrals we replace the sum by product and the product by raising to power of a function $F$ on $[a, b]$.

We give the following relation between Riemann integral and *integral: 
Proposition 1. If a positive function $F$ is Riemann integrable on $[a, b]$, then $F$ is *integrable on $[a, b]$ and

$$
\int_{a}^{b}(F(x))^{d x}=e^{\int_{a}^{b} \ln (F(x)) d x} .
$$

For further details of *integral reader can read [5].

Now we recall the concept of Interval-valued integral given by R. E. Moore in 28]. Let $F:[a, b] \rightarrow \mathbb{R}_{\mathcal{I}}$ be an interval-valued function such that $F(t)=[\underline{F}(t), \bar{F}(t)]$. The interval-valued Riemann integral of function $F$ is defined by

$$
\int_{a}^{b} F(x) d x=\int_{a}^{b}[\underline{F}(x), \bar{F}(x)] d x .
$$

Let's define interval-valued *integral or multiplicative integral $\left(I^{*} R\right)$ :

A function $F$ is said to be an interval-valued function of $t$ on $[a, b]$ if it assigns a nonempty interval to each $t \in[a, b]$

$$
F(t)=[\underline{F}(t), \bar{F}(t)] .
$$

A partition of $[a, b]$ is any finite ordered subset $\mathcal{P}$ having the form

$$
\mathcal{P}: a=t_{0}<t_{1}<\ldots<t_{n}=b .
$$

The mesh of a partition $\mathcal{P}$ is defined by

$$
\operatorname{mesh}(\mathcal{P})=\max \left\{t_{i}-t_{i-1}: i=1,2, \ldots, n\right\} .
$$

We denote by $\mathcal{P}([a, b])$ the set of all partition of $[a, b]$. Let $\mathcal{P}(\delta,[a, b])$ be the set of all $\mathcal{P}_{1} \in \mathcal{P}([a, b])$ such that $m e s h(\mathcal{P})<\delta$. Choose an arbitrary point $\xi_{i}$ in interval $\left[t_{i-1}, t_{i}\right], i=1,2, \ldots, n$ and we define the product

$$
P\left(F, \mathcal{P}_{1}, \delta\right)=\prod_{i=1}^{n} F\left(\xi_{i}\right)^{\left[t_{i}-t_{i-1}\right]}
$$

where $F:[a, b] \rightarrow \mathbb{R}_{\mathcal{I}}$ is a positive function. We call $P\left(F, \mathcal{P}_{1}, \delta\right)$ a Riemann product of $F$ corresponding to $\mathcal{P}_{1} \in \mathcal{P}(\delta,[a, b])$.

Definition 1. A positive function $F:[a, b] \rightarrow \mathbb{R}_{\mathcal{I}}$ is said to be integrable in multiplicative sense or *integrable ( $I^{*} R$ integrable) on $[a, b]$ if there exists $A \in \mathbb{R}_{I}$ such that, for each $\varepsilon>0$, there exists $\delta>0$ such that

$$
d\left(P\left(F, \mathcal{P}_{1}, \delta\right), A\right)<\varepsilon
$$

for every Riemann product $P$ of $F$ corresponding to each $\mathcal{P}_{1} \in \mathcal{P}(\delta,[a, b])$ and independent of choice of $\xi_{i} \in\left[t_{i-1}, t_{i}\right]$ for $1 \leq i \leq n$. In this case, $A$ is called the $I^{*} R$-integral of $F$ on $[a, b]$ and is denoted by

$$
A=\left(I^{*} R\right) \int_{a}^{b}(F(t))^{d t} .
$$


The collection of all functions that are $I^{*} R$ integrable on $[a, b]$ will be denote by $\mathcal{I}^{*} \mathcal{R}_{([a, b])}$.

The following theorem gives relation between $I^{*} R$-integral and multiplicative integral ( $I^{*}$-integral):

Theorem 1. Let $F:[a, b] \rightarrow \mathbb{R}_{\mathcal{I}}$ be a positive interval-valued function such that $F(t)=[\underline{F}(t), \bar{F}(t)] \in \mathcal{I}^{*} \mathcal{R}_{([a, b])}$ if and only if $\underline{F}(t), \bar{F}(t) \in \mathcal{I}_{([a, b])}^{*}$ and

$$
\left(I^{*} R\right) \int_{a}^{b}(F(t))^{d t}=\left[\left(I^{*}\right) \int_{a}^{b}(\underline{F}(t))^{d t},\left(I^{*}\right) \int_{a}^{b}(\bar{F}(t))^{d t}\right]
$$

where $\mathcal{I}_{([a, b])}^{*}$ denotes the all *integrable functions.

It is seen easily that if $F(t) \subseteq \mathcal{G}(t)$ for all $t \in[a, b]$, then $\left(I^{*} R\right) \int_{a}^{b}(F(t))^{d t} \subseteq$ $\left(I^{*} R\right) \int_{a}^{b}(\mathcal{G}(t))^{d t}$.

It is very easy to notice that if positive function $F$ is interval-valued integrable ( $I R$-integrable), then $F$ is $I^{*} R$ integrable and

$$
\left(I^{*} R\right) \int_{a}^{b}(F(t))^{d t}=e^{\int_{a}^{b}(\ln \circ F)(t) d t} .
$$

As we know that $\ln \circ F$ is $(I R)$ integrable on $[a, b]$ and continuity of the exponential we have

$$
P\left(F, \mathcal{P}_{1}, \delta\right)=e^{\sum_{i=1}^{n}\left(t_{i}-t_{i-1}\right)(\ln \circ F)\left(\xi_{i}\right)}
$$

imply the above statement and conversely, we have

$$
\int_{a}^{b} F(t) d t=\ln \int_{a}^{b}\left(e^{F(t)}\right)^{d t}
$$

Example 1. A positive interval-valued function $F:[1,2] \rightarrow \mathbb{R}_{I}$ be defined by

$$
F(t)=\left[t^{2}, e^{t^{2}}\right],
$$

then $F$ is interval-valued *integrable on $[1,2]$ and $\int_{1}^{2}\left(\left[t^{2}, e^{t^{2}}\right]\right)^{d t}=e^{\int_{1}^{2}\left[2 \ln t, t^{2}\right] d t}=$ [2.1651, 10.3119].

Now we give some properties of $*$ integral for interval valued functions. We consider $F$ and $\mathcal{G}$ are positive interval-valued functions then the following equalities hold:

(1) $\int_{a}^{b}\left(F(t)^{p}\right)^{d t}=\left(\int_{a}^{b}(F(t))^{d t}\right)^{p}$

(2) $\int_{a}^{b}(F(t) \mathcal{G}(t))^{d t}=\int_{a}^{b}(F(t))^{d t} \cdot \int_{a}^{b}(\mathcal{G}(t))^{d t}$

(3) $\int_{a}^{b}\left(\frac{F(t)}{\mathcal{G}(t)}\right)^{d t}=\frac{\int_{a}^{b}(F(t))^{d t}}{\int_{a}^{b}(\mathcal{G}(t))^{d t}}$ 
(4) $\int_{a}^{b}(F(t))^{d t}=\int_{a}^{c}(F(t))^{d t} \cdot \int_{c}^{b}(F(t))^{d t}$, where $a \leq c \leq b$.

Proof. Now we give the proofs of above properties.

(1) $\Longrightarrow$

$$
\begin{aligned}
\int_{a}^{b}\left(F(t)^{p}\right)^{d t} & =e^{\int_{a}^{b} \ln \left(F(t)^{p}\right) d t} \\
& =\left(e^{\int_{a}^{b} \ln (F(t)) d t}\right)^{p} \\
& =\left(\int_{a}^{b}(F(t))^{d t}\right)^{p}
\end{aligned}
$$

$(2) \Longrightarrow$

$$
\begin{aligned}
\int_{a}^{b}(F(t) \mathcal{G}(t))^{d t} & =e^{\int_{a}^{b} \ln (F(t) \mathcal{G}(t)) d t} \\
& =e^{\int_{a}^{b} \ln (F(t)) d t+\int_{a}^{b} \ln (\mathcal{G}(t)) d t} \\
& =\int_{a}^{b}(F(t))^{d t} \cdot \int_{a}^{b}(\mathcal{G}(t))^{d t}
\end{aligned}
$$

$(3) \Longrightarrow$

$$
\begin{aligned}
\int\left(\frac{F(t)}{\mathcal{G}(t)}\right)^{d t}= & e^{\int_{a}^{b} \ln \left(\frac{F(t)}{\mathcal{G}(t)}\right) d t} \\
= & e^{\int_{a}^{b} \ln (F(t)) d t-\int_{a}^{b} \ln (\mathcal{G}(t)) d t} \\
& \frac{\int_{a}^{b}(F(t))^{b}}{\int_{a}^{b}(\mathcal{G}(t))^{d t}}
\end{aligned}
$$

$(4) \Longrightarrow$

$$
\begin{aligned}
\int_{a}^{b}(F(t))^{d t} & =e^{\int_{a}^{b} \ln (F(t)) d t} \\
& =e^{\int_{a}^{c} \ln (F(t)) d t+\int_{c}^{d} \ln (F(t)) d t} \\
& =\int_{a}^{c}(F(t))^{d t} \cdot \int_{c}^{b}(F(t))^{d t}
\end{aligned}
$$

\section{Logarithmically Interval-valued Convex Functions}

In [42, Zhao et al. introduced a kind of interval-valued convex function as follows: 
Definition 2. Let $h:[c, d] \rightarrow \mathbb{R}$ be a non-negative function, $(0,1) \subseteq[c, d]$ and $h \neq 0$. We say that $F:[a, b] \rightarrow \mathbb{R}_{\mathcal{I}}^{+}$is an interval-valued $h$-convex function if for all $x, y \in[a, b]$ and $t \in(0,1)$, we have

$$
h(t) F(x)+h(1-t) F(y) \subseteq F(t x+(1-t) y) .
$$

$S X\left(h,[a, b], \mathbb{R}_{\mathcal{I}}^{+}\right)$will show the set of all interval-valued $h$-convex functions.

The usual notion of convex interval-valued function corresponds to relation 4.1 with $h(t)=t$ 35. Also, if $h(t)=t^{s}$ in 4.1], then Definition 2 gives the other interval-valued convex function defined by Breckner 6].

Definition 3. 30 Let $h:[c, d] \rightarrow \mathbb{R}$ be a non-negative function, $(0,1) \subseteq[c, d]$ and $h \neq 0$. A function $F:[a, b] \rightarrow(0, \infty)$ is called log-h-convex function, if

$$
F(t x+(1-t) y) \leq[F(x)]^{h(t)}[F(y)]^{h(1-t)},
$$

for all $x, y \in[a, b]$ and $t \in[0,1]$. For brevity, we can say that $F \in S X L\left[h,[a, b], \mathbb{R}_{I}^{+}\right]$ instead of logarithmically interval-valued- $h$-convex function.

In 20], Guo et al. gave the concept of interval-valued log- $h$-convex functions as follows:

Definition 4. Let $h:[c, d] \rightarrow \mathbb{R}$ be a non-negative function, $(0,1) \subseteq[c, d]$ and $h \neq 0$. A function $F:[a, b] \rightarrow \mathbb{R}_{I}^{+}$is said to be logarithmically interval-valued- $h$ convex function if for all $x, y \in[a, b]$ and $t \in[0,1]$, we have

$$
[F(x)]^{h(t)}[F(y)]^{h(1-t)} \subseteq F(t x+(1-t) y) .
$$

Remark 1. If we set $h(t)=t$ in Definition 4 , then we have new definition of intervalvalued convex function which is called log-interval-valued convex function.

Definition 5. A function $F:[a, b] \rightarrow \mathbb{R}_{I}^{+}$is said to be log-interval-valued convex function if for all $x, y \in[a, b]$ and $t \in[0,1]$, we have

$$
[F(x)]^{t}[F(y)]^{1-t} \subseteq F(t x+(1-t) y) .
$$

Remark 2. If we use $h(t)=t^{s}$ in Definition 4 then we have a new definition of interval-valued convex function which is called $s$-logarithmically interval-valued convex function.

Definition 6. A function $F:[a, b] \rightarrow \mathbb{R}_{I}^{+}$is said to be s-log-interval-valued convex function if for all $x, y \in[a, b], s \in[0,1]$ and $t \in[0,1]$, we have

$$
[F(x)]^{t^{s}}[F(y)]^{(1-t)^{s}} \subseteq F(t x+(1-t) y) .
$$

Remark 3. If we use $h(t)=1$ in Definition 4, then we have a new definition of interval-valued convex function which is called logarithmically interval-valued Pconvex function. 
Definition 7. A function $F:[a, b] \rightarrow \mathbb{R}_{I}^{+}$is said to be log-interval-valued P-convex function if for all $x, y \in[a, b]$, and $t \in[0,1]$, we have

$$
[F(x)][F(y)] \subseteq F(t x+(1-t) y) .
$$

Remark 4. If we put $h(t)=\frac{1}{t}$ in Definition 4, then we have a new definition of interval-valued convex function which is called logarithmically interval-valued Q-convex function.

Definition 8. A function $F:[a, b] \rightarrow \mathbb{R}_{I}^{+}$is said to be log-interval-valued Q-convex function if for all $x, y \in[a, b]$, and $t \in[0,1]$, we have

$$
[F(x)]^{\frac{1}{t}}[F(y)]^{\frac{1}{1-t}} \subseteq F(t x+(1-t) y) .
$$

Remark 5. If we put $h(t)=\frac{1}{t^{s}}$ in Definition 4 then we have a new definition of interval-valued convex function which is called s-logarithmically interval-valued Q-convex function.

Definition 9. A function $F:[a, b] \rightarrow \mathbb{R}_{I}^{+}$is said to be s-log-interval-valued Qconvex function if for all $x, y \in[a, b], s \in[0,1]$ and $t \in[0,1]$, we have

$$
[F(x)]^{\frac{1}{t^{s}}}[F(y)]^{\frac{1}{(1-t)^{s}}} \subseteq F(t x+(1-t) y) .
$$

Remark 6. If we put $h(t)=h\left(\frac{1}{2}\right)$ in Definition 4, then we have a new definition of interval-valued convex function which is called logarithmically interval-valued jensen type convex function.

Definition 10. A function $F:[a, b] \rightarrow \mathbb{R}_{I}^{+}$is said to be log-interval-valued jensen type convex function if for all $x, y \in[a, b]$ and $t \in[0,1]$, we have

$$
[F(x)]^{h\left(\frac{1}{2}\right)}[F(y)]^{h\left(\frac{1}{2}\right)} \subseteq F(t x+(1-t) y) .
$$

Proposition 2. If $F, \mathcal{G} \in S X L\left[h,[a, b], \mathbb{R}_{I}^{+}\right]$, then $F \mathcal{G} \in S X L\left[h,[a, b], \mathbb{R}_{I}^{+}\right]$.

\section{Hermite-Hadamard inequalities}

Theorem 2. Let $F:[a, b] \rightarrow \mathbb{R}_{\mathcal{I}}^{+}, h:[0,1] \rightarrow \mathbb{R}^{+}$and $h\left(\frac{1}{2}\right) \neq 0$. If $F \in$ $S X L\left(h,[a, b], \mathbb{R}_{\mathcal{I}}^{+}\right)$and $F \in \mathcal{I}^{*} \mathcal{R}_{([a, b])}$, then following double inequality holds:

$$
\left[F\left(\frac{a+b}{2}\right)\right]^{\frac{1}{2 h\left(\frac{1}{2}\right)}} \supseteq\left(\int_{a}^{b} F(x)^{d x}\right)^{\frac{1}{b-a}} \supseteq[F(a) F(b)]^{\int_{0}^{1} h(t) d t} .
$$

Proof. Since $F \in S X L\left(h,[a, b], \mathbb{R}_{\mathcal{I}}^{+}\right)$, then we have

$$
F\left(\frac{x+y}{2}\right) \supseteq[F(x)]^{h\left(\frac{1}{2}\right)}[F(y)]^{h\left(\frac{1}{2}\right)} .
$$

By setting $x=t a+(1-t) b$ and $y=t b+(1-t) a$, we get

$$
\ln F\left(\frac{a+b}{2}\right) \supseteq h\left(\frac{1}{2}\right)[\ln F(t a+(1-t) b)+\ln F(t b+(1-t) a)] .
$$


Integrating inequality $[5.2$ with respect to $t$ over $[0,1]$, we have

$$
\begin{aligned}
\ln F\left(\frac{a+b}{2}\right) \supseteq & h\left(\frac{1}{2}\right)\left[\int_{0}^{1} \ln F(t a+(1-t) b) d t\right. \\
& \left.+\int_{0}^{1} \ln F(t b+(1-t) a) d t\right]
\end{aligned}
$$

and by changing the variable of integration, we have

$$
\ln F\left(\frac{a+b}{2}\right) \supseteq \frac{2 h\left(\frac{1}{2}\right)}{b-a} \int_{a}^{b} \ln F(x) d x
$$

which implies that

$$
\ln \left[F\left(\frac{a+b}{2}\right)\right]^{\frac{1}{2 h\left(\frac{1}{2}\right)}} \supseteq \frac{1}{b-a} \int_{a}^{b} \ln F(x) d x .
$$

Hence

$$
\begin{aligned}
{\left[F\left(\frac{a+b}{2}\right)\right]^{\frac{1}{2 h\left(\frac{1}{2}\right)}} } & \supseteq\left(e^{\int_{a}^{b} \ln F(x) d x}\right)^{\frac{1}{b-a}} \\
& =\left(\int_{a}^{b}(F(x))^{d x}\right)^{\frac{1}{b-a}}
\end{aligned}
$$

which is the first inequality in (5.1).

Now we have to prove second inequality in 5.1), for this first we note that $F \in$ $S X L\left(h,[a, b], \mathbb{R}_{\mathcal{I}}^{+}\right)$, so we have

$$
\ln F(t a+(1-t) b) \supseteq h(t) \ln F(a)+h(1-t) \ln F(b) .
$$

Integrating inequality 5.3 with respect to $t$ over $[0,1]$ and change the variable of integration we have

$$
\begin{aligned}
\frac{1}{b-a} \int_{a}^{b} \ln F(x) d x & \supseteq[\ln F(a)+\ln F(b)] \int_{0}^{1} h(t) d t \\
& =\ln (F(a) F(b))^{\int_{0}^{1} h(t) d t}
\end{aligned}
$$

which implies that

$$
\left(e^{\int_{a}^{b} \ln F(x) d x}\right)^{\frac{1}{b-a}} \supseteq(F(a) F(b))^{\int_{0}^{1} h(t) d t} .
$$

Thus

$$
\left(\int_{a}^{b}(F(x))^{d x}\right)^{\frac{1}{b-a}} \supseteq(F(a) F(b))^{\int_{0}^{1} h(t) d t}
$$

which is the second inequality in (5.1).

The proof of the theorem is completed.

Remark 7. If $\underline{F}=\bar{F}$, then our Theorem 2 reduces to 30 , Theorem 3.1]. 
Corollary 1. Under the assumptions of Theorem 2, if we put $h(t)=t$, then we have following new Hermite-Hadamard inequality for log-interval-valued convex functions

$$
F\left(\frac{a+b}{2}\right) \supseteq\left(\int_{a}^{b}(F(x))^{d x}\right)^{\frac{1}{b-a}} \supseteq G(F(a), F(b))
$$

where $G(F(a), F(b))$ referes to the geometric mean of $F(a)$ and $F(b)$.

Example 2. A function $F:[x, y] \rightarrow \mathbb{R}_{I}^{+}$with $0<x<y$ is defined as

$$
F(x)=[\underline{F}(x), \bar{F}(x)]=\left[\frac{1}{x}, e^{x}-1\right],
$$

then $F \in S X L\left([a, b], \mathbb{R}_{\mathcal{I}}^{+}\right)$and we have

$$
\left(\int_{a}^{b}(F(x))^{d x}\right)^{\frac{1}{b-a}}=e^{\frac{1}{b-a}\left[\ln \left(\frac{b^{b}}{a^{a}}\right)^{-1}+1, \frac{b^{2}-a^{2}}{2}\right]} .
$$

On the other hand

$$
F\left(\frac{a+b}{2}\right)=\left[\frac{2}{a+b}, e^{\frac{a+b}{2}}-1\right]
$$

and

$$
\begin{aligned}
G(F(a), F(b)) & =\sqrt{F(a) F(b)} \\
& =\left[\sqrt{\frac{1}{a b}}, \sqrt{\left(e^{a}-1\right)\left(e^{b}-1\right)}\right] .
\end{aligned}
$$

Thus for all $a, b \in[x, y]$ inequalities (5.4) satisfies and we have

$$
\left[\frac{2}{a+b}, e^{\frac{a+b}{2}}-1\right] \supseteq e^{\frac{1}{b-a}\left[\ln \left(\frac{b^{b}}{a^{a}}\right)^{-1}+1, \frac{b^{2}-a^{2}}{2}\right]} \supseteq\left[\sqrt{\frac{1}{a b}}, \sqrt{\left(e^{a}-1\right)\left(e^{b}-1\right)}\right]
$$

for verification we suppose $a=1$ and $b=2$, then we have

$$
[0.6666,3.4816] \supseteq[0.6796,3.4260] \supseteq[0.7071,3.3131] .
$$

Remark 8. If $\underline{F}=\bar{F}$ in Corollary 1 , then we have inequality for log-convex function which can be found in 14, p. 197, (5.3)].

Corollary 2. Under the assumptions of Theorem 2, if we let $h(t)=t^{s}$, then we have a new inequality of s-logarithmically interval-valued convex functions

$$
\left[F\left(\frac{a+b}{2}\right)\right]^{2^{s-1}} \supseteq\left(\int_{a}^{b}(F(x))^{d x}\right)^{\frac{1}{b-a}} \supseteq[F(a) F(b)]^{\frac{1}{s+1}} .
$$

Remark 9. If $\underline{F}=\bar{F}$ in Corollary 2 then Corollary 2 reduces to 30 , Corollary 3.3]. 
Corollary 3. Under the conditions of Theorem 2, if we use $h(t)=1$, then we have a new inequality for the logarithmically interval-valued $P$-convex functions

$$
\sqrt{F\left(\frac{a+b}{2}\right)} \supseteq\left(\int_{a}^{b}(F(x))^{d x}\right)^{\frac{1}{b-a}} \supseteq F(a) F(b) .
$$

Remark 10. If $\underline{F}=\bar{F}$ in Corollary 3 , then Corollary 3 reduces to 30 , Corollary 3.4].

Corollary 4. Under the assumptions of Theorem 2, if we set $h(t)=t^{-1}$ in first inequality, then we have following inequality for the logarithmically interval-valued $Q$-convex functions

$$
\left[F\left(\frac{a+b}{2}\right)\right]^{\frac{1}{4}} \supseteq\left(\int_{a}^{b}(F(x))^{d x}\right)^{\frac{1}{b-a}} .
$$

Remark 11. If $\underline{F}=\bar{F}$ in Corollary 4 , Then Corollary 4 reduces to 30 , Corollary $3.5]$.

Corollary 5. Under the conditions of Theorem 2, if suppose $h(t)=t^{-s}$ in first inequality, then we have new inequality for s-logarithmically interval-valued $Q$-convex functions

$$
\left[F\left(\frac{a+b}{2}\right)\right]^{\frac{1}{2^{s+1}}} \supseteq\left(\int_{a}^{b}(F(x))^{d x}\right)^{\frac{1}{b-a}} .
$$

Remark 12. If $\underline{F}=\bar{F}$ in Corollary 5 , then we have following new inequality for the $s$-logarithmically $Q$-convex functions

$$
\left[F\left(\frac{a+b}{2}\right)\right]^{\frac{1}{2^{s+1}}} \leq\left(\int_{a}^{b}(F(x))^{d x}\right)^{\frac{1}{b-a}} .
$$

Theorem 3. Let $F, \mathcal{G}:[a, b] \rightarrow \mathbb{R}_{\mathcal{I}}^{+}, h:[0,1] \rightarrow \mathbb{R}^{+}$and $h\left(\frac{1}{2}\right) \neq 0$. If $F, \mathcal{G} \in$ $S X L\left(h,[a, b], \mathbb{R}_{\mathcal{I}}^{+}\right)$and $F, \mathcal{G} \in \mathcal{I}^{*} \mathcal{R}_{([a, b])}$, then following double inequality holds:

$$
\begin{aligned}
{\left[F\left(\frac{a+b}{2}\right) \mathcal{G}\left(\frac{a+b}{2}\right)\right]^{\frac{1}{2 h\left(\frac{1}{2}\right)}} } & \supseteq\left(\int_{a}^{b} F(x)^{d x} \cdot \int_{a}^{b} \mathcal{G}(x)^{d x}\right)^{\frac{1}{b-a}} \\
& \supseteq[F(a) F(b) \mathcal{G}(a) \mathcal{G}(b)]^{\int_{0}^{1} h(t) d t} .
\end{aligned}
$$

Proof. Since $F, \mathcal{G} \in S X L\left(h,[a, b], \mathbb{R}_{\mathcal{I}}^{+}\right)$, so $F$ and $\mathcal{G}$ can be written as

$$
F\left(\frac{x+y}{2}\right) \supseteq[F(x)]^{h\left(\frac{1}{2}\right)}[F(y)]^{h\left(\frac{1}{2}\right)}
$$

and

$$
\mathcal{G}\left(\frac{x+y}{2}\right) \supseteq[\mathcal{G}(x)]^{h\left(\frac{1}{2}\right)}[\mathcal{G}(y)]^{h\left(\frac{1}{2}\right)} .
$$


By setting $x=t a+(1-t) b$ and $y=t b+(1-t) a$ in (5.6) and (5.7), we have

$$
F\left(\frac{a+b}{2}\right) \supseteq[F(t a+(1-t) b)]^{h\left(\frac{1}{2}\right)}[F(t b+(1-t) a)]^{h\left(\frac{1}{2}\right)}
$$

and

$$
\mathcal{G}\left(\frac{a+b}{2}\right) \supseteq[\mathcal{G}(t a+(1-t) b)]^{h\left(\frac{1}{2}\right)}[\mathcal{G}(t b+(1-t) a)]^{h\left(\frac{1}{2}\right)} .
$$

From (5.8) and (5.9), we have

$$
\begin{aligned}
& \ln F\left(\frac{a+b}{2}\right) \mathcal{G}\left(\frac{a+b}{2}\right) \\
\supseteq & h\left(\frac{1}{2}\right)[\ln F(t a+(1-t) b)+\ln F(t b+(1-t) a) \\
& +\ln \mathcal{G}(t a+(1-t) b)+\ln \mathcal{G}(t b+(1-t) a)]
\end{aligned}
$$

and integrating (5.10) with respect to $t$ over $[0,1]$, we have

$$
\begin{aligned}
& \ln F\left(\frac{a+b}{2}\right) \mathcal{G}\left(\frac{a+b}{2}\right) \\
\supseteq & h\left(\frac{1}{2}\right)\left[\int_{0}^{1} \ln F(t a+(1-t) b) d t+\int_{0}^{1} \ln F(t b+(1-t) a) d t\right. \\
& \left.+\int_{0}^{1} \ln \mathcal{G}(t a+(1-t) b) d t+\int_{0}^{1} \ln \mathcal{G}(t b+(1-t) a) d t\right] .
\end{aligned}
$$

By changing the variable in last inequality, we have

$$
\frac{1}{2 h\left(\frac{1}{2}\right)} \ln F\left(\frac{a+b}{2}\right) \mathcal{G}\left(\frac{a+b}{2}\right) \supseteq \frac{1}{b-a}\left[\int_{a}^{b} \ln F(x) d x+\int_{a}^{b} \ln \mathcal{G}(x) d x\right]
$$

which implies that

$$
\left[F\left(\frac{a+b}{2}\right) \mathcal{G}\left(\frac{a+b}{2}\right)\right]^{\frac{1}{2 h\left(\frac{1}{2}\right)}} \supseteq\left(e^{\int_{a}^{b} \ln F(x) d x+\int_{a}^{b} \ln \mathcal{G}(x) d x}\right)^{\frac{1}{b-a}} .
$$

Hence

$$
\left[F\left(\frac{a+b}{2}\right) \mathcal{G}\left(\frac{a+b}{2}\right)\right]^{\frac{1}{2 h\left(\frac{1}{2}\right)}} \supseteq\left(\int_{a}^{b} F(x)^{d x} \cdot \int_{a}^{b} \mathcal{G}(x)^{d x}\right)^{\frac{1}{b-a}}
$$

which is the first inequality in (5.5).

To prove the second inequality in $(5.5)$, first we note that $F, \mathcal{G} \in S X L\left(h,[a, b], \mathbb{R}_{\mathcal{I}}^{+}\right)$, so we have

$$
F(t a+(1-t) b) \supseteq[F(a)]^{h(t)}[F(b)]^{h(1-t)}
$$

and

$$
\mathcal{G}(t a+(1-t) b) \supseteq[\mathcal{G}(a)]^{h(t)}[\mathcal{G}(b)]^{h(1-t)} .
$$


From 5.12 and (5.13), we have

$$
\begin{aligned}
& \ln F(t a+(1-t) b) \mathcal{G}(t a+(1-t) b) \\
\supseteq & h(t)[\ln F(a)+\ln \mathcal{G}(a)]+h(1-t)[\ln F(b)+\ln \mathcal{G}(b)]
\end{aligned}
$$

and integrating inequality 5.14 with respect to $t$ over $[0,1]$, we get

$$
\begin{aligned}
& \int_{0}^{1} \ln F(t a+(1-t) b) \mathcal{G}(t a+(1-t) b) \\
\supseteq \quad & {[\ln F(a)+\ln \mathcal{G}(a)] \int_{0}^{1} h(t) d t } \\
+ & {[\ln F(b)+\ln \mathcal{G}(b)] \int_{0}^{1} h(1-t) d t . }
\end{aligned}
$$

By changing the variable of integration, we have

$$
\frac{1}{b-a} \int_{0}^{1} \ln F(x) \mathcal{G}(x) d x \supseteq \ln [F(a) F(b) \mathcal{G}(a) \mathcal{G}(b)]^{\int_{0}^{1} h(t) d t}
$$

which implies that

$$
\left(e^{\int_{a}^{b} \ln F(x) d x+\int_{a}^{b} \ln \mathcal{G}(x) d x}\right)^{\frac{1}{b-a}} \supseteq[F(a) F(b) \mathcal{G}(a) \mathcal{G}(b)]^{\int_{0}^{1} h(t) d t} .
$$

Thus

$$
\left(\int_{a}^{b} F(x)^{d x} \cdot \int_{a}^{b} \mathcal{G}(x)^{d x}\right)^{\frac{1}{b-a}} \supseteq[F(a) F(b) \mathcal{G}(a) \mathcal{G}(b)]^{\int_{0}^{1} h(t) d t}
$$

which is the second inequality in 5.5.

The proof of theorem is completed.

Corollary 6. If we use $h(t)=t$ in Theorem 3, then following new inequalities for logarithmically interval-valued convex functions hold:

$$
\begin{aligned}
F\left(\frac{a+b}{2}\right) \mathcal{G}\left(\frac{a+b}{2}\right) & \supseteq\left(\int_{a}^{b} F(x)^{d x} \cdot \int_{a}^{b} \mathcal{G}(x)^{d x}\right)^{\frac{1}{b-a}} \\
& \supseteq G(F(a), F(b)) \cdot G(\mathcal{G}(a), \mathcal{G}(b))
\end{aligned}
$$

where $G(.,$.$) referes to the geometric mean.$

Remark 13. If $\underline{F}=\bar{F}$ in Corollary 6, then Corollary 6 reduces to 3 , Theorem 7].

Corollary 7. Under the assumptions of Theorem 3, if we let $h(t)=t^{s}$, then following inequalities for the s-logarithmically interval-valued convex functions hold:

$$
\left[F\left(\frac{a+b}{2}\right) \mathcal{G}\left(\frac{a+b}{2}\right)\right]^{2^{s-1}} \supseteq\left(\int_{a}^{b} F(x)^{d x} \cdot \int_{a}^{b} \mathcal{G}(x)^{d x}\right)^{\frac{1}{b-a}}
$$




$$
\supseteq[F(a) F(b) \mathcal{G}(a) \mathcal{G}(b)]^{\frac{1}{s+1}} .
$$

Corollary 8. Under the conditions of Theorem 3, if we choose $h(t)=1$, then we have following inequalities for the logarithmically P-convex functions

$$
\begin{aligned}
F\left(\frac{a+b}{2}\right) \mathcal{G}\left(\frac{a+b}{2}\right) & \supseteq\left(\int_{a}^{b} F(x)^{d x} \cdot \int_{a}^{b} \mathcal{G}(x)^{d x}\right)^{\frac{2}{b-a}} \\
& \supseteq[F(a) F(b) \mathcal{G}(a) \mathcal{G}(b)]^{2} .
\end{aligned}
$$

Corollary 9. If we put $h(t)=t^{-1}$ in first inequality of Theorem 3, then we have following inequalities for the logarithmically interval-valued $Q$-convex functions

$$
\left[F\left(\frac{a+b}{2}\right) \mathcal{G}\left(\frac{a+b}{2}\right)\right]^{\frac{1}{4}} \supseteq\left(\int_{a}^{b} F(x)^{d x} \cdot \int_{a}^{b} \mathcal{G}(x)^{d x}\right)^{\frac{1}{b-a}} .
$$

Corollary 10. Under the assumptions of Theorem 3, if we set $h(t)=t^{-s}$ in first inequality, then following inequalities for s-logarithmically interval-valued $Q$-convex functions hold

$$
\left[F\left(\frac{a+b}{2}\right) \mathcal{G}\left(\frac{a+b}{2}\right)\right]^{\frac{1}{2^{s+1}}} \supseteq\left(\int_{a}^{b} F(x)^{d x} \cdot \int_{a}^{b} \mathcal{G}(x)^{d x}\right)^{\frac{1}{b-a}} .
$$

Theorem 4. Let $F:[a, b] \rightarrow \mathbb{R}_{\mathcal{I}}^{+}, h:[0,1] \rightarrow \mathbb{R}^{+}$and $h\left(\frac{1}{2}\right) \neq 0$. If $F \in$ $S X L\left(h,[a, b], \mathbb{R}_{\mathcal{I}}^{+}\right)$and $F \in \mathcal{I}^{*} \mathcal{R}_{([a, b])}$, then following inequalities hold:

$$
\begin{aligned}
& \left(F\left(\frac{a+b}{2}\right)\right)^{\frac{1}{2 h^{2}\left(\frac{1}{2}\right)}} \supseteq \Delta_{1}^{\frac{1}{4 h\left(\frac{1}{2}\right)}} \\
& \supseteq\left(\int_{a}^{b} F(x)^{d x}\right)^{\frac{1}{b-a}} \\
& \supseteq \Delta_{2}^{\frac{1}{2} \int_{0}^{1} h(t) d t} \\
& \supseteq(F(a) F(b))^{\left[\frac{1}{2}+h\left(\frac{1}{2}\right)\right] \int_{0}^{1} h(t) d t},
\end{aligned}
$$

where

$$
\begin{aligned}
& \Delta_{1}=F\left(\frac{3 a+b}{4}\right) F\left(\frac{a+3 b}{4}\right) \\
& \Delta_{2}=F(a) F(b) F^{2}\left(\frac{a+b}{2}\right) .
\end{aligned}
$$

Proof. Since $F \in S X L\left(h,[a, b], \mathbb{R}_{\mathcal{I}}^{+}\right)$then for $t \in\left[a, \frac{a+b}{2}\right]$, we have

$$
\ln F\left(\frac{3 a+b}{4}\right)
$$




$$
\begin{aligned}
& =\ln F\left(\frac{t a+(1-t) \frac{a+b}{2}}{2}+\frac{(1-t) a+t \frac{a+b}{2}}{2}\right) \\
& \supseteq \ln F\left(\frac{t a+(1-t) \frac{a+b}{2}}{2}\right)^{h\left(\frac{1}{2}\right)} F\left(\frac{(1-t) a+t \frac{a+b}{2}}{2}\right)^{h\left(\frac{1}{2}\right)} \\
& =h\left(\frac{1}{2}\right)\left[\ln F\left(\frac{t a+(1-t) \frac{a+b}{2}}{2}\right)+\ln F\left(\frac{(1-t) a+t \frac{a+b}{2}}{2}\right)\right] .
\end{aligned}
$$

Integrating inequality 5.15 with respect to $t$ over $[0,1]$, we have

$$
\begin{aligned}
\ln F\left(\frac{3 a+b}{2}\right) \supseteq & h\left(\frac{1}{2}\right)\left[\int_{0}^{1} \ln F\left(\frac{t a+(1-t) \frac{a+b}{2}}{2}\right) d t\right. \\
& \left.+\int_{0}^{1} \ln F\left(\frac{(1-t) a+t \frac{a+b}{2}}{2}\right) d t\right]
\end{aligned}
$$

and by changing the variable of integration, we have

$$
\ln \left(F\left(\frac{3 a+b}{4}\right)\right)^{\frac{1}{4 h\left(\frac{1}{2}\right)}} \supseteq \frac{1}{b-a} \int_{a}^{\frac{a+b}{2}} \ln F(x) d x
$$

which implies that

$$
\left(F\left(\frac{3 a+b}{4}\right)\right)^{\frac{1}{4 h\left(\frac{1}{2}\right)}} \supseteq\left(\int_{a}^{\frac{a+b}{2}} F(x)^{d x}\right)^{\frac{1}{b-a}} .
$$

Similarly, we have

$$
\left(F\left(\frac{a+3 b}{4}\right)\right)^{\frac{1}{4 h\left(\frac{1}{2}\right)}} \supseteq\left(\int_{\frac{a+b}{2}}^{b} F(x)^{d x}\right)^{\frac{1}{b-a}} .
$$

Multiplying (5.16) and (5.17), we have

$$
\Delta_{1}^{\frac{1}{4 h\left(\frac{1}{2}\right)}}=\left(F\left(\frac{3 a+b}{4}\right) F\left(\frac{a+3 b}{4}\right)\right)^{\frac{1}{4 h\left(\frac{1}{2}\right)}} \supseteq\left(\int_{a}^{b} F(x)^{d x}\right)^{\frac{1}{b-a}} .
$$

Now from Theorem 2, one has

$$
\begin{aligned}
\left(F\left(\frac{a+b}{2}\right)\right)^{\frac{1}{4 h^{2}\left(\frac{1}{2}\right)}} & =\left[F\left(\frac{1}{2} \frac{3 a+b}{4}+\frac{1}{2} \frac{a+3 b}{4}\right)\right]^{\frac{1}{4 h^{2}\left(\frac{1}{2}\right)}} \\
& \supseteq\left[F\left(\frac{3 a+b}{4}\right)^{h\left(\frac{1}{2}\right)} F\left(\frac{a+3 b}{4}\right)^{h\left(\frac{1}{2}\right)}\right]^{\frac{1}{4 h^{2}\left(\frac{1}{2}\right)}} \\
& \supseteq \Delta_{1}^{\frac{1}{4 h\left(\frac{1}{2}\right)}}
\end{aligned}
$$




$$
\begin{aligned}
& \supseteq\left(\int_{a}^{b} F(x)^{d x}\right)^{\frac{1}{b-a}} \\
& \supseteq\left(F(a) F(b) F^{2}\left(\frac{a+b}{2}\right)\right)^{\frac{1}{2} \int_{0}^{1} h(t) d t} \\
& =\Delta_{2}^{\frac{1}{2} \int_{0}^{1} h(t) d t} \\
& \supseteq\left([F(a) F(b)]^{\frac{1}{2}}[F(a) F(b)]^{h\left(\frac{1}{2}\right)}\right)^{\int_{0}^{1} h(t) d t} \\
& =(F(a) F(b))^{\left[\frac{1}{2}+h\left(\frac{1}{2}\right)\right] \int_{0}^{1} h(t) d t}
\end{aligned}
$$

which completes the proof.

Corollary 11. Under the assumptions of Theorem (4), if we set $h(t)=t$ in Theorem 4, then following inequalities for the logarithmically interval-valued convex functions hold

$$
\begin{aligned}
\left(F\left(\frac{a+b}{2}\right)\right)^{2} & \supseteq \sqrt{\Delta_{1}} \supseteq\left(\int_{a}^{b} F(x)^{d x}\right)^{\frac{1}{b-a}} \\
& \supseteq \Delta_{2}^{\frac{1}{4}} \supseteq G(F(a), F(b))
\end{aligned}
$$

where $G(.,$.$) referes to the geometric mean.$

Example 3. A function $F:[x, y] \rightarrow \mathbb{R}_{I}^{+}$with $0<x<y$ is defined as

$$
F(x)=[\underline{F}(x), \bar{F}(x)]=\left[\frac{1}{x}, e^{x}-1\right],
$$

then $F \in S X L\left([a, b], \mathbb{R}_{\mathcal{I}}^{+}\right)$and we have

$$
\left(\int_{a}^{b}(F(x))^{d x}\right)^{\frac{1}{b-a}}=e^{\frac{1}{b-a}\left[\ln \left(\frac{b^{b}}{a^{a}}\right)^{-1}+1, \frac{b^{2}-a^{2}}{2}\right]} .
$$

On the other hand

$$
\begin{aligned}
F\left(\frac{a+b}{2}\right) & =\left[\frac{2}{a+b}, e^{\frac{a+b}{2}}-1\right] \\
\Delta_{1} & =\left[\frac{16}{(3 a+b)(a+3 b)},\left(e^{\frac{3 a+b}{4}}-1\right)\left(e^{\frac{a+3 b}{4}}-1\right)\right] \\
\Delta_{2} & =\left[\frac{1}{a b},\left(e^{a}-1\right)\left(e^{b}-1\right)\right]\left[\frac{2}{a+b}, e^{\frac{a+b}{2}}-1\right]^{2}
\end{aligned}
$$

and

$$
G(F(a), F(b))=\sqrt{F(a) F(b)}
$$




$$
=\left[\sqrt{\frac{1}{a b}}, \sqrt{\left(e^{a}-1\right)\left(e^{b}-1\right)}\right] .
$$

Thus for all $a, b \in[x, y]$ inequalities (5.18) satisfies and we have

$$
\begin{aligned}
{\left[\frac{2}{a+b}, e^{\frac{a+b}{2}}-1\right]^{2} } & \supseteq \sqrt{\left[\frac{16}{(3 a+b)(a+3 b)},\left(e^{\frac{3 a+b}{4}}-1\right)\left(e^{\frac{a+3 b}{4}}-1\right)\right]} \\
& \supseteq e^{\frac{1}{b-a}\left[\ln \left(\frac{b^{b}}{a^{a}}\right)^{-1}+1, \frac{b^{2}-a^{2}}{2}\right]} \\
& \supseteq\left(\left[\frac{1}{a b},\left(e^{a}-1\right)\left(e^{b}-1\right)\right]\left[\frac{2}{a+b}, e^{\frac{a+b}{2}}-1\right]^{2}\right)^{\frac{1}{4}} \\
& \supseteq\left[\sqrt{\frac{1}{a b}}, \sqrt{\left(e^{a}-1\right)\left(e^{b}-1\right)}\right]
\end{aligned}
$$

for verification we suppose $a=1$ and $b=2$, then we have

$$
\begin{aligned}
{[0.4444,12.1215] } & \supseteq[0.6761,3.4409] \\
& \supseteq[0.6796,3.4260] \\
& \supseteq[0.6865,3.3963] \\
& \supseteq[0.7071,3.3131] .
\end{aligned}
$$

Corollary 12. Under the assumptions of Theorem 4. if we let $h(t)=t^{s}$, then following inequalities for the s-logarithmically interval-valued convex functions hold:

$$
\begin{aligned}
\left(F\left(\frac{a+b}{2}\right)\right)^{2^{2 s-1}} & \supseteq \Delta_{1}^{2^{s-2}} \supseteq\left(\int_{a}^{b} F(x)^{d x}\right)^{\frac{1}{b-a}} \\
& \supseteq \Delta_{2}^{\frac{1}{2(s+1)}} \supseteq(F(a) F(b))^{\frac{1}{s+1}\left[\frac{1}{2}+\frac{1}{2^{s}}\right]} .
\end{aligned}
$$

Corollary 13. Under the conditions of Theorem 4, if we choose $h(t)=1$, then we have following inequalities for the logarithmically $P$-convex functions

$$
\begin{aligned}
\sqrt{F\left(\frac{a+b}{2}\right)} & \supseteq \Delta_{1}^{\frac{1}{4}} \supseteq\left(\int_{a}^{b} F(x)^{d x}\right)^{\frac{1}{b-a}} \\
& \supseteq \sqrt{\Delta_{2}} \supseteq(F(a) F(b))^{\frac{3}{2}} .
\end{aligned}
$$

Corollary 14. If we put $h(t)=t^{-1}$ in first two inequalities of Theorem 4, then we have following inequalities for the logarithmically interval-valued $Q$-convex functions

$$
\left(F\left(\frac{a+b}{2}\right)\right)^{\frac{1}{8}} \supseteq \Delta_{1}^{\frac{1}{8}} \supseteq\left(\int_{a}^{b} F(x)^{d x}\right)^{\frac{1}{b-a}} .
$$


Corollary 15. Under the assumptions of Theorem 4, if we set $h(t)=t^{-s}$ in first inequality, then following inequalities for s-logarithmically interval-valued $Q$-convex functions hold

$$
\left(F\left(\frac{a+b}{2}\right)\right)^{\frac{1}{2^{2 s+1}}} \supseteq \Delta_{1}^{\frac{1}{22 s+1}} \supseteq\left(\int_{a}^{b} F(x)^{d x}\right)^{\frac{1}{b-a}} .
$$

\section{Conclusion}

In this paper, authors define multiplicative integral for the interval-valued functions and derived some new Hermite-Hadamard and related inequalities for logarithmically interval-valued $h$-convex functions by utilizing our newly define integral. Authors also gave some more new results in the special cases of our main results. Interested readers can obtain more results by using the notions used in this paper. The results in this paper can be a new contribution in the field of Hermite-Hadamard integral inequalities.

Acknowledgment. This work is partially supported by National Natural Science Foundation of China (No. 11971241).

\section{REFERENCES}

[1] Ali, M. A., Abbas, M., Budak, H., Kashuri, A., Some new Hermite-Hadamard integral inequalities in multiplicative calculus, TWMS Journal of Applied and Engineering Mathematics (2020, In Press).

[2] Ali, M. A., Abbas, M., Zafar, A. A., On some Hermite-Hadamard integral inequalities in multiplicative calculus, Journal of Inequalities and Special Functions, 10 (1) (2019), 111122.

[3] Ali, M. A., Abbas, M., Zhang, Z., Sial, I. B., Arif, R., On integral inequalities for product and quotient of two multiplicatively convex functions, Asian Research Journal of Mathematics (2019), 1-11.

[4] Aubin, J.-P., Cellina, A., Differential inclusions: set-valued maps and viability theory, Springer Science \& Business Media, Springer-Verlage Berlin Heidelberg New York Tokyo, 2012.

[5] Bashirov, A. E., Kurpınar, E. M., Özyapıcı, A., Multiplicative calculus and its applications, Journal of Mathematical Analysis and Applications, 337 (1) (2008), 36-48.

[6] Breckner, W. W., Continuity of generalized convex and generalized concave set-valued functions, Rev. Anal. Numér. Théor. Approx., 22 (1) (1993), 39-51.

[7] Budak, H., Ali, M. A., Tarhanaci, M., Some new quantum Hermite-Hadamard-like inequalities for coordinated convex functions, Journal of Optimization Theory and Applications, 186 (3) (2020), 899-910.

[8] Budak, H., Erden, S., Ali, M. A., Simpson and Newton type inequalities for convex functions via newly defined quantum integrals, Mathematical Methods in the Applied Sciences (2020).

[9] Chalco-Cano, Y., Flores-Franulic, A., Román-Flores, H., Ostrowski type inequalities for interval-valued functions using generalized hukuhara derivative, Computational $\&$ Applied Mathematics, 31 (3) (2012).

[10] Chalco-Cano, Y., Lodwick, W. A., Condori-Equice, W., Ostrowski type inequalities and applications in numerical integration for interval-valued functions, Soft Computing, 19 (11) (2015), 3293-3300. 
[11] Chen, F., A note on Hermite-Hadamard inequalities for products of convex functions., Journal of Applied Mathematics (2013).

[12] Costa, T., Jensen's inequality type integral for fuzzy-interval-valued functions, Fuzzy Sets and Systems, 327 (2017), 31-47.

[13] Costa, T., Román-Flores, H., Some integral inequalities for fuzzy-interval-valued functions, Information Sciences, 420 (2017), 110-125.

[14] Dragomir, S., Pearce, C., Selected topics on Hermite-Hadamard inequalities and applications, rgmia monographs, victoria university, 2000, ONLINE: http://rgmia. vu. edu. au/monographs (2004).

[15] Dragomir, S., Pecaric, J., Persson, L.-E., Some inequalities of Hadamard type, Soochow J. Math, 21 (3) (1995), 335-341.

[16] Dragomir, S. S., Two mappings in connection to Hadamard's inequalities, Journal of Mathematical Analysis and Applications, 167 (1) (1992), 49-56.

[17] Dragomir, S. S., Inequalities of Hermite-Hadamard type for h-convex functions on linear spaces, Proyecciones (Antofagasta), 34 (4) (2015), 323-341.

[18] Ertugral, F., Sarikaya, M. Z., Simpson type integral inequalities for generalized fractional integral, Revista de la Real Academia de Ciencias Exactas, Fisicas y Naturales. Serie A. Matematicas, 113 (4) (2019), 3115-3124.

[19] Flores-Franulič, A., Chalco-Cano, Y., Román-Flores, H., An ostrowski type inequality for interval-valued functions, In 2013 Joint IFSA World Congress and NAFIPS Annual Meeting (IFSA/NAFIPS) (2013), IEEE, pp. 1459-1462.

[20] Guo, Y., Ye, G., Zhao, D., Liu, W., Some integral inequalities for log- $h$-convex interval-valued functions, IEEE Access, 7 (2019), 86739-86745.

[21] Kara, H., Ali, M. A., Budak, H., Hermite-Hadamard-type inequalities for interval-valued coordinated convex functions involving generalized fractional integrals, Mathematical Methods in the Applied Sciences (2020).

[22] Lupulescu, V., Fractional calculus for interval-valued functions, Fuzzy Sets and Systems, 265 (2015), 63-85.

[23] Markov, S., Calculus for interval functions of a real variable, Computing, 22 (4) (1979), $325-337$.

[24] Markov, S., On the algebraic properties of convex bodies and some applications, Journal of convex analysis, 7 (1) (2000), 129-166.

[25] Mitroi, F.-C., Nikodem, K., Wasowicz, S., Hermite-Hadamard inequalities for convex setvalued functions, Demonstratio Mathematica, 46 (4) (2013), 655-662.

[26] Mohammed, P., Some new Hermite-Hadamard type inequalities for mt-convex functions on differentiable coordinates, Journal of King Saud University-Science, 30 (2) (2018), 258-262.

[27] Moore, R. E., Interval analysis, Prentice-Hall, Englewood Cliffs, 1966.

[28] Moore, R. E., Kearfott, R. B., Cloud, M. J., Introduction to interval analysis, Siam, Philadelphia, P. A., 2009

[29] Nikodem, K., Sanchez, J. L., Sanchez, L., Jensen and Hermite-Hadamard inequalities for strongly convex set-valued maps, Mathematica Aeterna, 4 (8) (2014), 979-987.

[30] Noor, M. A., Qi, F., Awan, M. U., Some Hermite-Hadamard type inequalities for log-h-convex functions, Analysis, 33 (4) (2013), 367-375.

[31] Pachpatte, B., On some inequalities for convex functions, RGMIA Res. Rep. Coll, 6 (1) (2003), 1-9.

[32] Peajcariaac, J. E., Tong, Y. L., Convex functions, partial orderings, and statistical applications, Academic Press, Bostan SanDiego New York London Sydney Tokyo Toronto, 1992.

[33] Román-Flores, H., Chalco-Cano, Y., Lodwick, W., Some integral inequalities for intervalvalued functions, Computational and Applied Mathematics, 37 (2) (2018), 1306-1318. 
[34] Román-Flores, H., Chalco-Cano, Y., Silva, G. N., A note on gronwall type inequality for interval-valued functions, In 2013 Joint IFSA World Congress and NAFIPS Annual Meeting (IFSA/NAFIPS) (2013), IEEE, pp. 1455-1458.

[35] Sadowska, E., Hadamard inequality and a refinement of Jensen inequality for set valued functions, Results in Mathematics, 32 (3-4) (1997), 332-337.

[36] Sarikaya, M. Z., Yildirim, H., On generalization of the riesz potential, Indian Jour. of Math. and Mathematical Sci, 3 (2) (2007), 231-235.

[37] Tseng, K.-L., Hwang, S.-R., New Hermite-Hadamard-type inequalities and their applications, Filomat, 30 (14) (2016), 3667-3680.

[38] Vivas-Cortez, M., Aamir Ali, M., Kashuri, A., Bashir Sial, I., Zhang, Z., Some new Newton's type integral inequalities for co-ordinated convex functions in quantum calculus, Symmetry, $12(9)(2020), 1476$.

[39] Wang, J., Li, X., Zhu, C., et al., Refinements of Hermite-Hadamard type inequalities involving fractional integrals, Bulletin of the Belgian Mathematical Society-Simon Stevin, 20 (4) (2013), 655-666.

[40] Zhao, D., Ali, M. A., Kashuri, A., Budak, H., Generalized fractional integral inequalities of Hermite-Hadamard type for harmonically convex functions, Advances in Difference Equations, 2020 (1) (2020), 1-14.

[41] Zhao, D., Ali, M. A., Murtaza, G., Zhang, Z., On the Hermite-Hadamard inequalities for interval-valued coordinated convex functions, Advances in Difference Equations, 2020, 570 (2020).

[42] Zhao, D., An, T., Ye, G., Liu, W., New Jensen and Hermite-Hadamard type inequalities for h-convex interval-valued functions, Journal of Inequalities and Applications, 2018 (1) (2018), 302 . 